\title{
Nail Syndrome Patella: Needs and Obligation to Renal Surveillance
}

\section{Boudghene Stambouli 0* and Zoulikha $\mathrm{H}$}

Department of Dermatology, Aboubakr Belkaid University- Hospital, Algeria

*Corresponding author: Prof. Omar Boudghene Stambouli, MD, Head of Department of Dermatology, Faculty of Medicine, University Aboubakr Belkaid, 13000, Algeria, Tel: +213658366871; Fax: 21343267270, E-mail: omar_stambouli@hotmail.com

\author{
Case Report \\ Volume 2 Issue 3
}

Received Date: August 19, 2017

Published Date: September 11, 2017

DOI: $10.23880 /$ cdoaj-16000132

\section{Abstract}

The "Nail-Patella syndrome" or onycho-osteodysplasia is transmitted as an autosomal dominant pleiotropic whose manifestations suggest an abnormality primary connective tissue. The disease has been described in all populations. It is characterized by dystrophic nails, fingers and toes (80 to 90\%), absence or hypoplasia of the ball joints, elbows dysplasia and exostosis of the iliac wings (30 to $70 \%$ of cases). Nephropathy is inconsistent with type of proteinuria sometimes nephrotic syndrome, hematuria (half the cases). Approximately $30 \%$ of patients progress to ESRD at an average age of 33. Objective: We report the observation of two different families

Observation: Nail Patella Syndrome (NPS) is a rare genetic disease, estimated at 22 cases / million in contrast to the poverty of familial cases reported in the literature impact. It is transmitted as an autosomal dominant trait with variable expression. Anomalies are located on the 9q34 genes LMXMB position are similar to those of ABO blood groups and adenylate cyclase and which encode a protein belonging to the family of LIM- homeobox domain, involved in limb morphogenesis and the anterior chamber of the eye, the maturation of renal podocytes increased collagen type I synthesis. It was suggested that the coexistence of two allelic mutations of which would be responsible for the form with nephropathy and the other form without nephropathy.

Methods: In fact, 80 to 90 percent of patients have onycho - skeletal abnormalities from birth .In our patients, the nail pterygium was reported at birth and orthopedic symptoms, confirmed by the very rich radiological exploration in both observations. During the SNP renal disease is objectified in half of cases among both men and women. In the first family were not noted or renal abnormalities in the father or to his daughter when we have objectified proteinuria in the second family. Ocular involvement is absent in both cases. Our first observation is characterized by the predominance of onycho skeletal involvement.

Results: This reinforces the hypothesis not responsible for renal disease allelic mutation. Scarf and coll.ont mentioned the existence of a new type of pediatric renal impairment where the nail and bone is absent. With reference to this entity, we can only strengthen suggestion that the mutation on a particular allele will be responsible for renal disease or not. 
Conclusion: The development of means including immune histochemical diagnostic and genetic genital will in the near future to determine the risk of developing since renal impairment was observed that the collagen fibrils are a constant manifestation of the SNP and are also observed in patients with renal impairment is not clinically detectable.

Keywords: Nail; Nephropathy; Syndrome Patella

\section{Introduction}

The syndrome "Nail-Patella" or onycho-osteodysplasia is transmitted in the autosomal dominant, pleiotropic mode whose manifestations suggest an anomaly primary connective tissue [1-7]. The disease has been described in all populations.

It is characterized by dystrophy of the nails, fingers and toes (80 to $90 \%$ ) (Figure 1), absence or hypoplasia of the

kneecaps, elbow dysplasia and exostosis of the iliac wings (30 70\% of cases) (Figures 2 \& 3). Nephropathy is inconstant to type of proteinuria sometimes nephrotic syndrome, hematuria (1/2 of cases). About $30 \%$ of patients progress to end-stage renal disease at an average age of 33 years.

It combines:
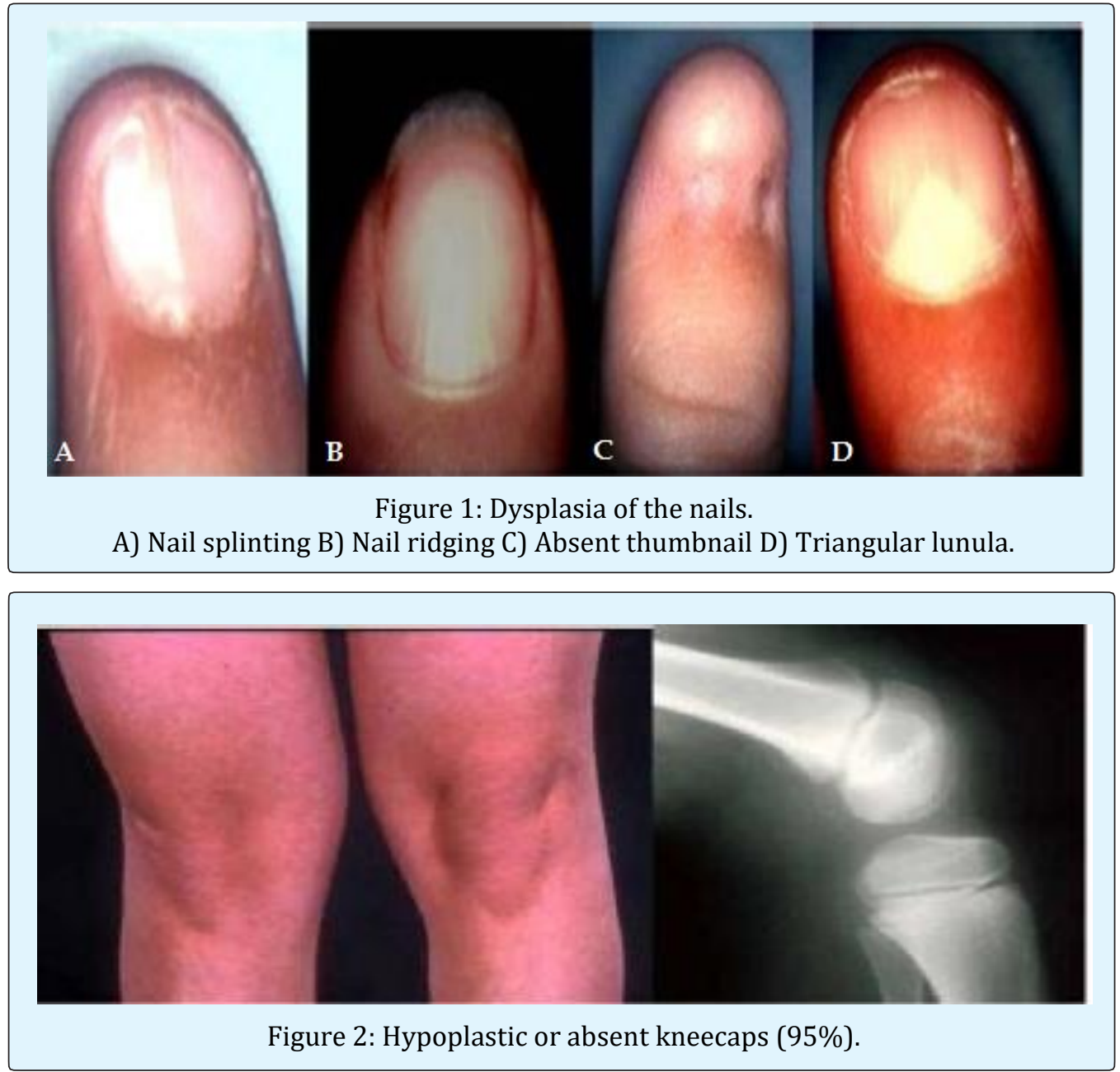

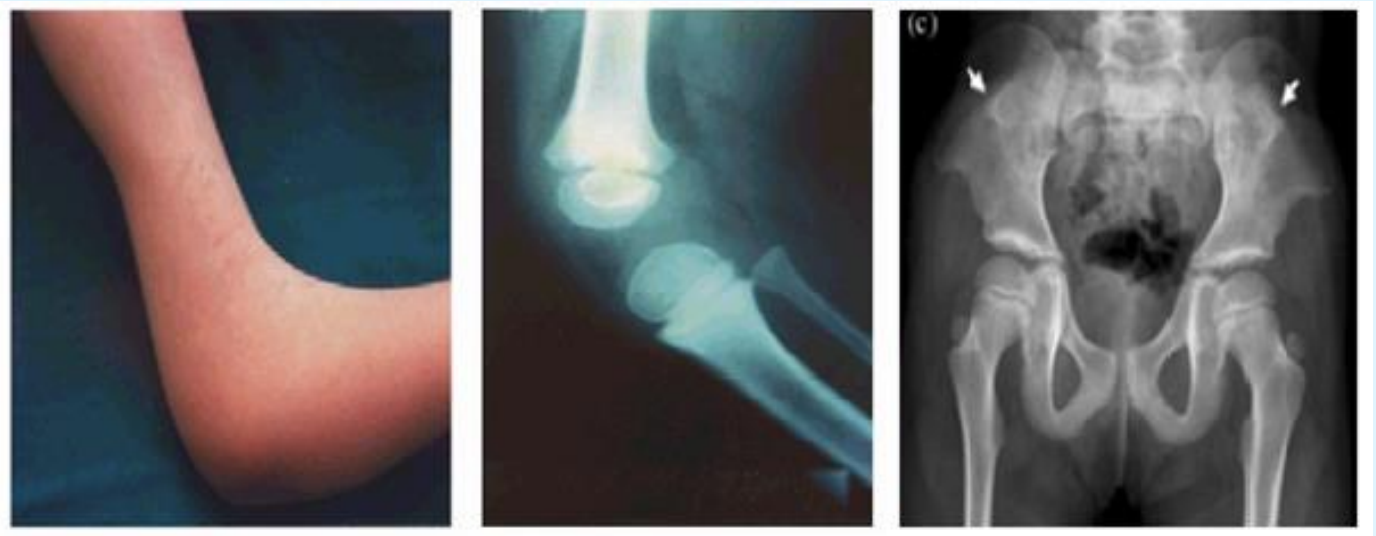

Figure 3: Elbow dysplasia (94\%) and exostosis of iliacwings (70\%).

Ocular involvement (glaucoma, ocular hypertension, etc.) is observed in approximately one third of the patients. Neurosensory deafness may be present. Glomerular nephropathy (40-50\%)

- It translates into: proteinuria, A nephrotic syndrome, hematuria HTA.

- Evolving towards IRT (30\%)

- MBG abnormalities

- Irregular thickening

- ME: Presence of fibrillar collagen

- IHC: Presence of type III collagen

\section{Etiology}

The disease gene was located on the distal portion of the long arm of chromosome 9 at $9 q 34$.

It has recently been demonstrated that LMXIB regulates the expression of alpha-3 and alpha-4 chains of type IV collagen in MBG. We report the observation of two different families.

\section{Observation No. 1}

We report the case of an Algerian family with several members (the father and three children) with predominant onycho-skeletal involvement.

Mrs. F. I, 40 years old, is the eldest daughter of a sibling of four, presents: (Figure 4)

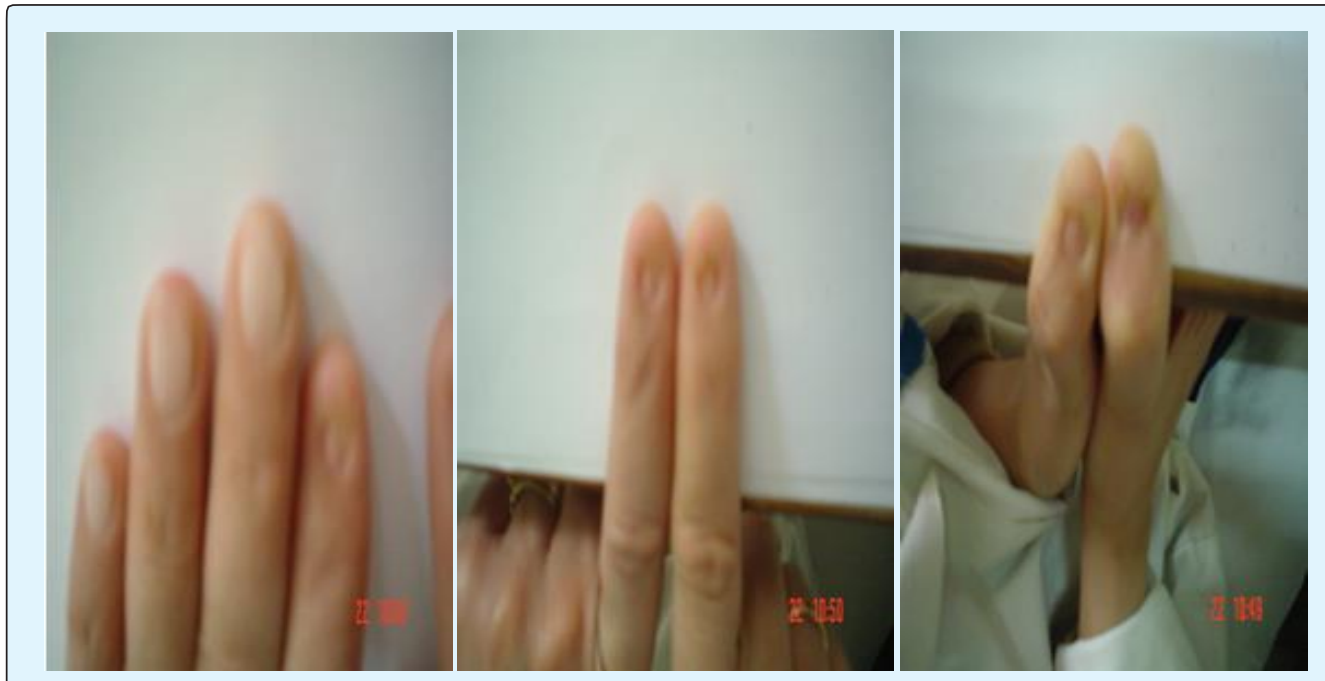

Figure 4: Pterygium nail.

On the orthopedic plane, the patient presents a waddling walk, with a limitation of the extension of the upper limbs and the lower left limb (Figures 5-7). 


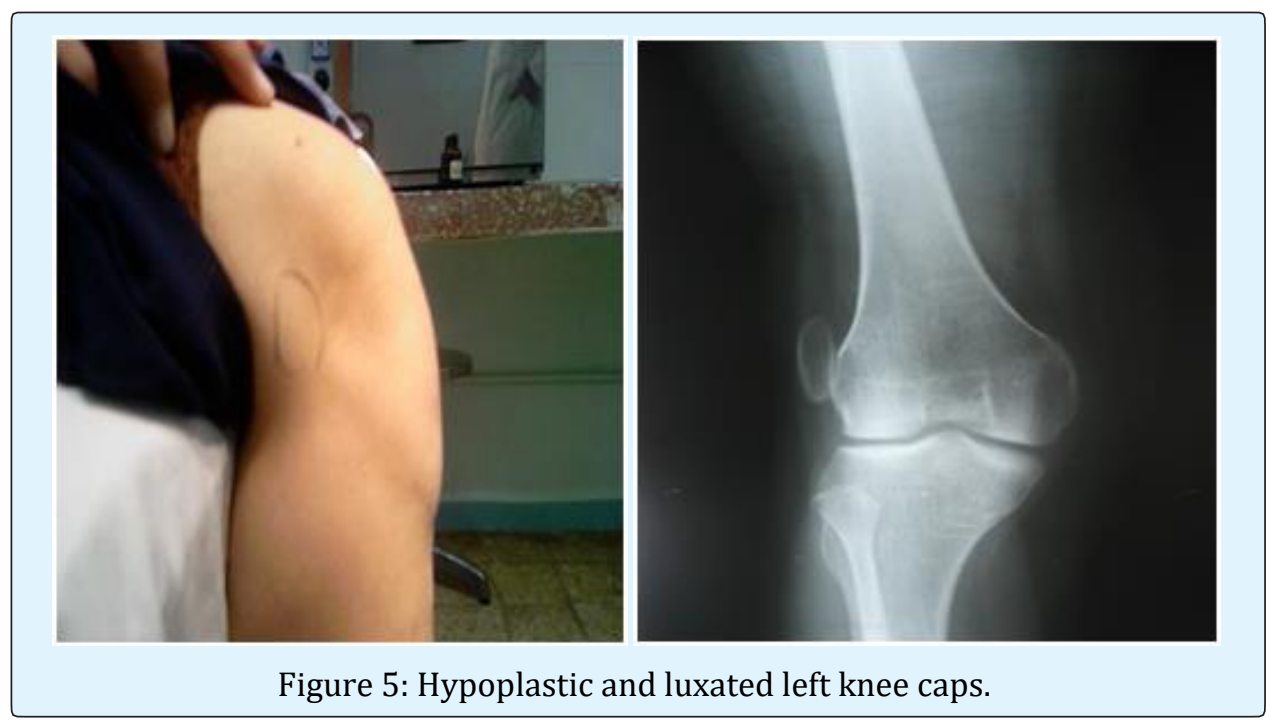

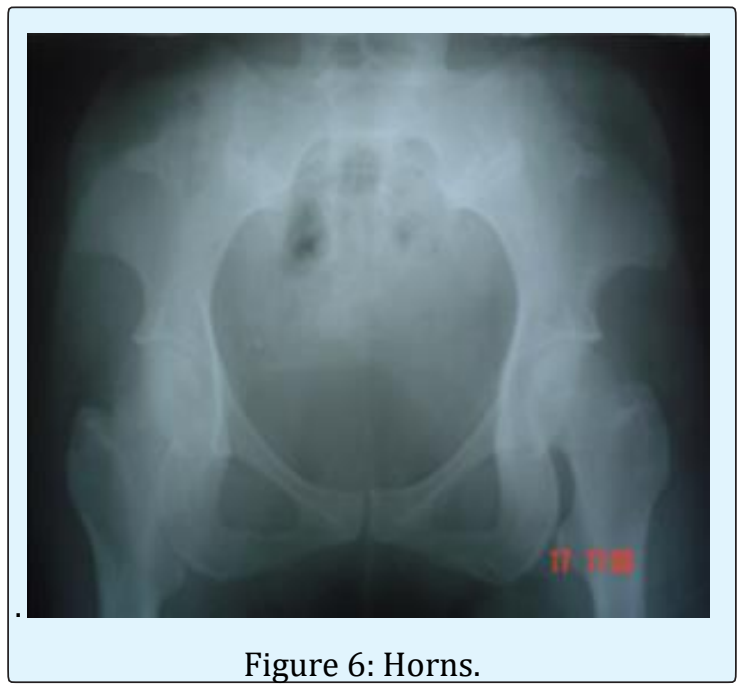

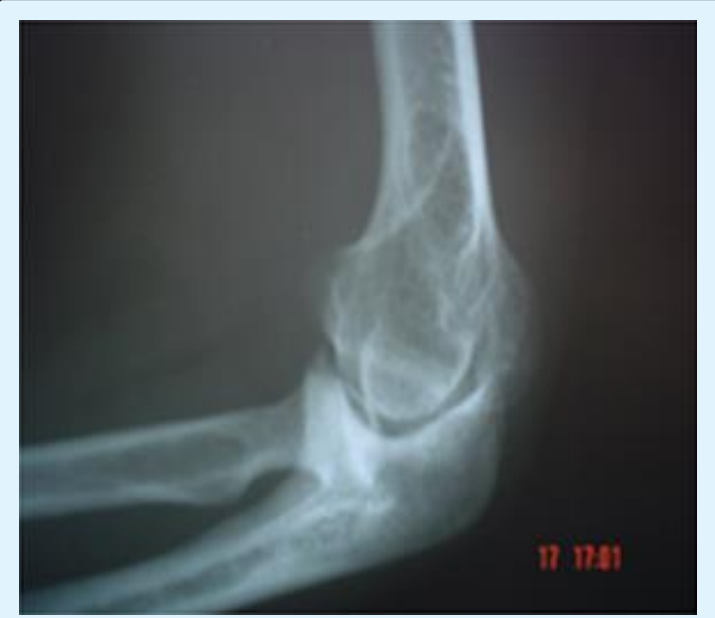

Figure 7: Subluxation of the radial head.
- Ophthalmologic examination is normal.

- Renal, biological and radiological examination revealed no abnormalities.

The father: F.A, aged 70, presents the same nail symptomatology with effacement of the folds of the dorsal aspect of the auriculars (Figure 8).

- The biological renal balance (urea, creatinine, proteinuria) is normal

- The patient refused radiological exploration

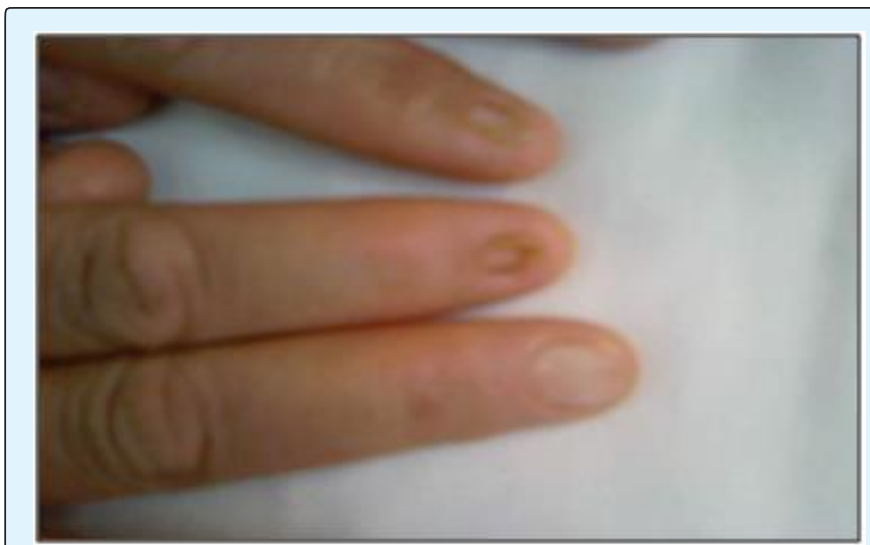

Figure 8: Nail pterygium in the father.

For the other two cases, the family describes the same nail anomalies in the brother and sister aged 37 and 30, who are currently living abroad.

\section{Observation No. 2}

Our second observation concerns a 29-year-old woman from a non-consanguineous marriage with a family 
history: a deficit in elbow extension at grandmother -She presents: (Figures 9 \&10).
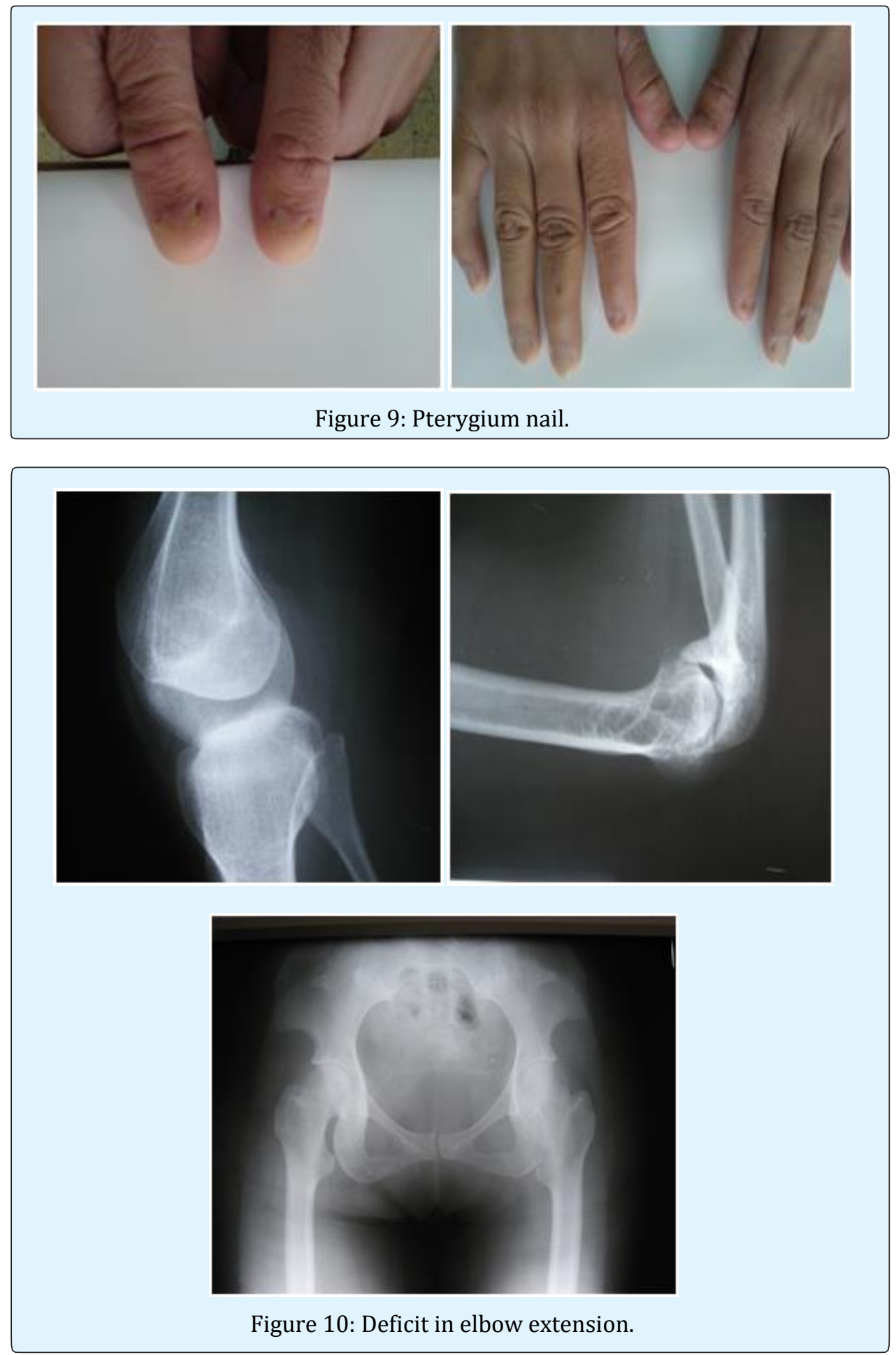


\section{Clinical Dermatology Open Access Journal}

\section{Discussion}

The nail patella syndrome (PNS) is a rare disease with an estimated incidence of 22 per million in contrast to the poverty of familial cases reported in the literature [3].

SNP is a dominant autosomal genetic pathology of variable expression, secondary to abnormalities of the LMXMB genes, located in 9q34, genes close to those of the ABO blood groups and the adenylcyclase, encoding a protein belonging to the family of LIM-omeodomain proteins, intervening on the morphogenesis of the limbs, on the maturation of the renal podocytes with increased synthesis of collagen type I, as well as on the morphogenesis of the structures of the anterior chamber of the eye [4]. Two allelic mutations have been suggested to coexist, one of which is responsible for the form with nephropathy and the other for the form without nephropathy [2].

Indeed, eighty to 90 percent of patients have onychoskeletal abnormalities from birth [3].

In our patients, the nail pterygium was reported from birth as well as the confirmed orthopedic symptomatology radiological exploration very rich in our first observation [3].

In the SNP, renal involvement was observed in half of the cases in both men and women. In our family there were no kidney abnormalities either in the father or in his daughter.

There is no ocular anomaly. The observations reported in this family are characterized by the predominance of onycho-skeletal involvement.

This supports the hypothesis of non-responsible allelic mutation of renal involvement. Frohlich SJ, et al mentioned the existence of a new type of pediatric nephropathy where nail and bone involvement is absent [6]. By referring to this entity, we can only reinforce the suggestion that the mutation on this or that allele would be responsible for kidney damage or not [1,5-7].

\section{Conclusion}

In the near future, the development of diagnostic tools, in particular immuno-histochemical and geni-genetic, will determine the risk of developing renal damage.

Indeed, it has been observed that collagen fibrils have been constantly present in the heart of the SNP and are also observed in patients whose renal involvement is not clinically detectable.

At present, prenatal diagnosis is possible because the mutant gene is detected and can be done by amniocentesis, and this prenatal diagnosis allows for genetic counseling.

\section{References}

1. Avermaete A, Altmeyer P, Bacharach-Buhles M (2001) Skin changes in dialysis patients: a review. Nephrol Dial Transplant 16(12): 2293-2296.

2. Meyrier A, Rizzo R, Gubler MC (1990) The nail-patella syndrome. A review. J Nephrol 2: 133-140.

3. Guidera KJ, Satterwhite Y, Ogden JA, Pugh L, Ganey T (1991) Nail Patella Syndrome: A Review of 44 Orthopaedic Patients. J Pediatr Orthop 11(6): $737-$ 742.

4. Lucas GL, Opitz JM (1966) The nail-patella syndrome. Clinical and genetic aspects of 5 kindreds with 38 affected family members. J Pediatr 68: 273-288.

5. Cohen AH, Adler SG (1994) Nail Patella Syndrome. In: Tisher CC, Brenner BM (Eds.), Renal pathology with clinical and functional correlations. Lippincott, Philadelphia.

6. Frohlich SJ (2002) Ophthalmology 99: 281.

7. Gubler MC, Dommergues JP, Foulard M, Bensman A, Leroy JP, et al. (1993) Collagene type III glomerulopathy: A new type of hereditary nephropathy. Pediatr Nephrol 7(4): 354-360. 